\title{
Power Delivery and Locomotion of Untethered Microactuators
}

\author{
Bruce Randall Donald, Member, IEEE, Christopher G. Levey, Associate Member, IEEE, \\ Craig D. McGray, Student Member, IEEE, Daniela Rus, Member, IEEE, and Mike Sinclair
}

\begin{abstract}
The ability for a device to locomote freely on a surface requires the ability to deliver power in a way that does not restrain the device's motion. This paper presents a MEMS actuator that operates free of any physically restraining tethers. We show how a capacitive coupling can be used to deliver power to untethered MEMS devices, independently of the position and orientation of those devices. Then, we provide a simple mechanical release process for detaching these MEMS devices from the fabrication substrate once chemical processing is complete. To produce these untethered microactuators in a batch-compatible manner while leveraging existing MEMS infrastructure, we have devised a novel postprocessing sequence for a standard MEMS multiproject wafer process. Through the use of this sequence, we show how to add, post hoc, a layer of dielectric between two previously deposited polysilicon films. We have demonstrated the effectiveness of these techniques through the successful fabrication and operation of untethered scratch drive actuators. Locomotion of these actuators is controlled by frequency modulation, and the devices achieve maximum speeds of over $1.5 \mathrm{~mm} / \mathrm{s}$.

[1034]
\end{abstract}

Index Terms-Capacitive coupling, microlocomotion, microrobotics, scratch drive actuators, untethered microactuators.

\section{INTRODUCTION}

W HILE the field of MEMS has produced a wide variety of innovative microactuators, considerably less research has been conducted on the possibility of autonomous locomotion at the microscale. Accordingly, though there are many actuators with dimensions expressible in tens of microns, the smallest existing self-contained locomotive platforms are in the millimeter to centimeter range [1]-[8]. As the size of these locomotion platforms decreases, the problem of power delivery requires novel solutions. This paper presents a power delivery mechanism for unrestrained actuators that are $40-100 \mu \mathrm{m}$ on a side. The actuators require no fixed connection to the substrate, and can operate at arbitrary position and orientation. Because device position and orientation are not restricted, this power

Manuscript received April 11, 2003; revised August 6, 2003. This work was supported by Department of Justice under Contract 2000-DT-CX-K001, a NASA Space-grant, National Science Foundation Grants EIA-0202789, EIA-9901589, IIS-9906790, EIA-0102710, EIA-0102712, EIA-9818299, EIA-9802 068, and IIS-9912193, and Microsoft Research. This paper was presented in part at IEEE MEMS 2003. Subject Editor C.-J. Kim.

B. R. Donald is with the Computer Science Department and the Dartmouth Chemistry Department, Dartmouth College, Hanover, NH 03755 USA (e-mail brd@cs.dartmouth.edu).

C. G. Levey is with the Dartmouth Thayer School of Engineering, Dartmouth College, Hanover, NH 03755 USA

C. D. McGray and D. Rus are with the Dartmouth Computer Science Department, Dartmouth College, Hanover, NH 03755 USA

M. Sinclair is with Microsoft Research, Redmond, WA 98052 USA.

Digital Object Identifier 10.1109/JMEMS.2003.821468 delivery mechanism is appropriate for fully two-dimensional $(x, y)$ and $(x, y, \theta)$ locomotive platforms. Fig. 1 shows the role of this mechanism in the progression toward locomotive platforms at the sub-100- $\mu \mathrm{m}$ scale.

Previous work has identified numerous applications for miniature and microscale locomotive platforms operating either as remote-operated vehicles or, with on-board control systems, as microrobots. These application areas include manipulation and assembly of hybrid microsystems [4], [9]-[13]; security and surveillance [4], [6]; exploration of hazardous environments [6]; and biomedical research [6], [10]. The possibility of untethered two-dimensional microactuators and microrobots with side lengths less than $100 \mu \mathrm{m}$ opens the door to many additional applications. For example, the planning and control of micropushing strategies could build on the mechanics of planar pushing manipulation [14] to extend existing distributed, multirobot pushing protocols [15]. As another example, in meso-scale self-assembly (e.g., [16]), the assembly constituents have traditionally been passive participants in the complex dynamical system that drives the self-assembly process. Consequently, to date, the components of a self-assembled system have been more like manipulanda, and less like robots. Microrobots at the $100 \mu \mathrm{m}$ scale would enable a self-assembly paradigm in which the pieces of a self-assembling system could be active participants in their own assembly, analogous to biological self-assembling systems. Applications are foreseen in microscale self-reconfiguring robotics [17]-[22] and in MEMS infosecurity self-assembly [23], [24], where autonomous locomotion of microdevices is a primary requirement. While for all these applications a number of technical and engineering challenges remain, in each case the capability of unrestrained, untethered locomotion serves as an important enabling technology.

Previous work on the power delivery problem has produced a number of different approaches. Energy has been provided to actuators through vibration [8], through photo-thermal transduction [1], [2], through inductive coupling [25], and electrically through gold bonding wire [4]-[6]. These approaches produced locomotive platforms with sizes between $1.5 \mathrm{~mm}$ and $3 \mathrm{~cm}$. The present paper proposes the use of a capacitive coupling for electrostatic power delivery, that allows fabrication and control of untethered actuators of less than $80 \mu \mathrm{m}$ in length, requiring a minimum of assembly, all of which can be automated on-chip. These new actuators have demonstrated the ability to assist in their own assembly, to push small objects, and to achieve operating speeds in excess of $1.5 \mathrm{~mm} / \mathrm{s}$ with step sizes less than $50 \mathrm{~nm}$. 


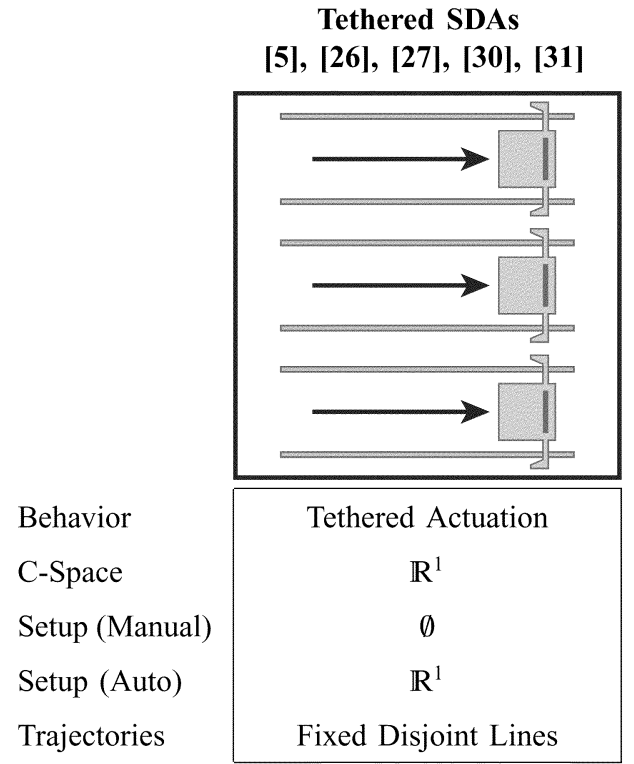

(a)
Untethered SDAs

[This Paper]

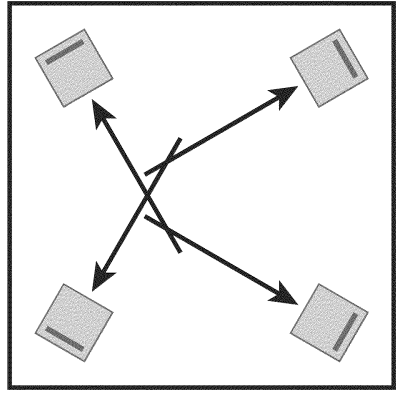

Untethered Locomotion

$\mathbb{R}^{1}$

$\mathbb{R}^{2} \times S^{1}$

$\mathbb{R}^{1}$

Arbitrary Lines

(b)
Steerable Microactuators [Future Work]

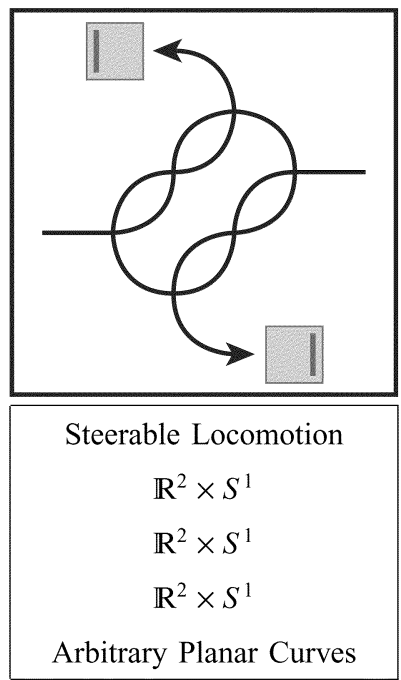

(c)

Fig. 1 Progress towards to fully two-dimensional (2-D) MEMS locomotive platforms. C-space (configuration space [38]) reports the total degrees of freedom (DOF) of the device motion. Setup reports the possible DOF to initialize the device's initial pose (position and orientation) prior to the motion either manually with a pair of microprobes (Мапиal), or in an automated fashion (Auto). The three classes of device are shown. Panel (a) characterizes the behavior of previous tethered scratch drive actuators (SDAs). The paths of these devices are constrained to fixed lines [26], [27], [30], [31] or circles [5], [12] that map onto $\mathbb{R}^{1}$ in configuration space. Panel (b) describes the untethered actuators presented in the current paper. These new devices also operate in the configuration $\mathbb{R}^{1}$, but can be manually initialized in the space $\mathbb{R}^{2} \times S^{1}$, where $\mathbb{R}^{2}$ is the Euclidean plane of the substrate, and $S^{1}$ is the group of 2-D rotations corresponding to the device's orientation. Hence, these untethered devices can move along arbitrary lines in the plane (as opposed to fixed paths, as in tethered SDAs [5], [26], [27], [30], [31]). Furthermore, while tethered SDAs are confined to disjoint lines, untethered SDAs can move along lines that overlap. Panel (c) characterizes the behavior of a (hypothetical) steerable microlocomotive platform. The untethered power delivery mechanism described in this paper enables the freedom of movement required for microdevices capable of the motions shown in panels (b) and (c).

\section{BACKGROUND-TETHERED SCRATCH DRIVE ACTUATORS}

A scratch drive [5], [26]-[31] is a type of direct-drive actuator that operates through electrostatic attraction. It is composed of a thin polysilicon plate with a bushing at the front end. The plate is typically in the range of $80 \mu \mathrm{m}$ on a side, and 1-2 $\mu \mathrm{m}$ thick. The bushing height is typically in the $1-2 \mu \mathrm{m}$ range. The plate is electrically connected to a voltage source through the same spring tether, rotor, or rail that provides it with physical connection to the substrate. The substrate is grounded, and insulated from the scratch drive by a thin layer of dielectric.

The scratch drive operates as shown in Fig. 2. When a voltage is applied between the polysilicon plate and the substrate beneath it, the plate is drawn down into contact with the dielectric layer. Since the front of the plate is supported by the bushing, strain energy is stored in the plate, and the edge of the bushing is pushed forward. When the voltage is removed, the strain is released and the scratch drive plate snaps back to its original shape, slightly in front of where it began. When a periodic pulse is applied, this cycle is continuously repeated, and the scratch drive moves forward in a stepwise manner.

The typical average step size achieved by a scratch drive actuator is around $30 \mathrm{~nm}$. This makes the scratch drive an ideal actuation mechanism for thin film microrobots. It allows for high precision of movement, while providing the possibility of fast speeds when the actuators are driven at high frequencies.

The problem with using scratch drives as the actuation system for a thin-film microrobot is that the power delivery mechanisms previously developed for SDAs rely on a fixed track along which the device travels. This track is defined by a rigid tether to a rotor
[5], [26], by a flexible connection to an anchor [27], [30], or by a sliding contact with a rail [26], [31]. To allow for motion in any arbitrary direction, a power delivery mechanism that does not constrain the motion of the device is required. This paper introduces such a mechanism, and describes its use in the first untethered scratch drive actuator.

\section{Power Delivery Mechanism}

One way to deliver power without restraining device motion is through a capacitive coupling with the substrate. This type of power delivery mechanism has been successfully used in the development of gas-lubricated micromotors [32] and has been proposed for use in flying insect-model microrobots [7].

To use capacitively-coupled power for fully $2-\mathrm{D}(x, y, \theta)$ locomotive platforms, the operating environment must be structured so that a constant voltage is maintained as the actuator changes its position and orientation. To do this, we cover the surface of the substrate with a sequence of insulated electrodes, as shown in Figs. 2 and 3.

When power and ground are applied to adjacent electrodes, an untethered scratch drive actuator placed in any orientation on these electrodes forms the capacitive circuit shown in Fig. 2. The potential applied to the scratch drive plate is the potential on the wire between the two capacitors in this circuit:

$$
V_{\text {plate }}=\frac{V_{1} C_{1}+V_{2} C_{2}}{C_{1}+C_{2}}
$$

where $V_{1}$ and $V_{2}$ are the applied voltages, and $C_{1}$ and $C_{2}$, are the capacitances. 


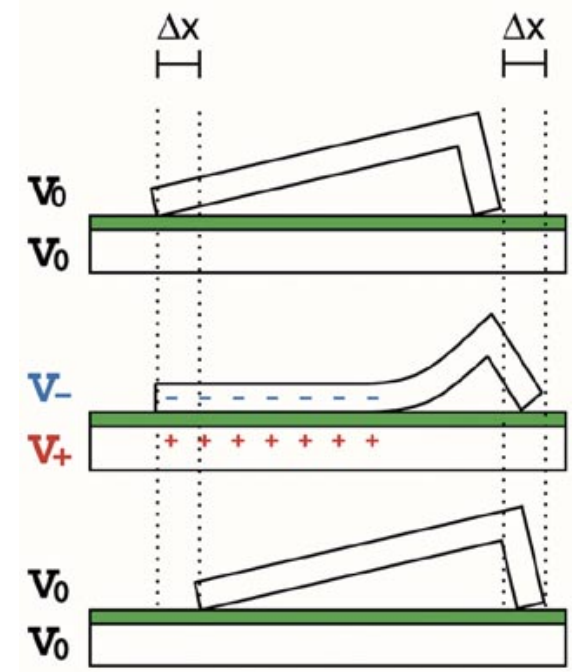

(a)

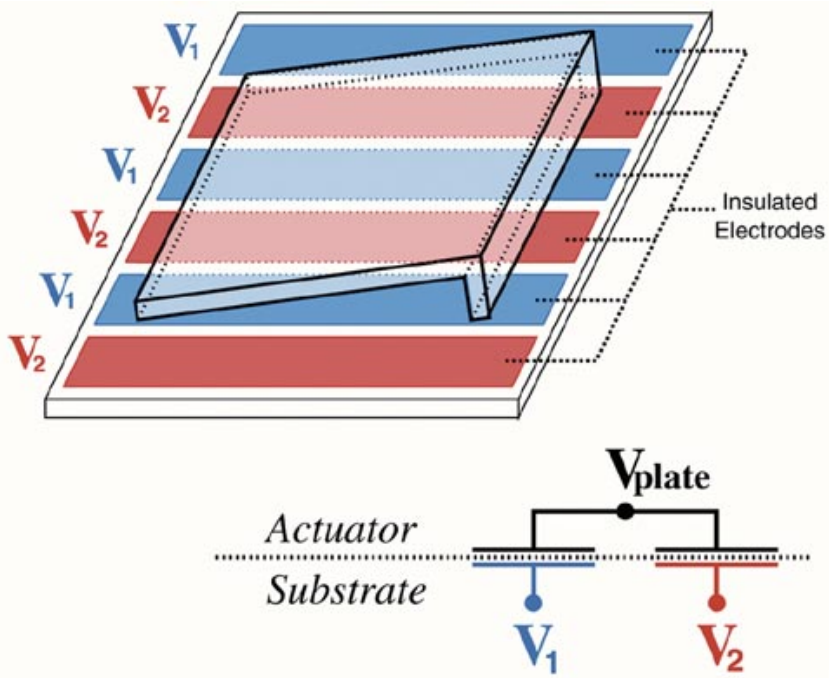

(b)

Fig. 2 (a) Schematic of the operation of a tethered scratch drive actuator [26], [27]. (b) Schematic of a capacitively coupled power delivery mechanism for untethered scratch drives. The potential induced on the actuator $V_{\text {plate }}$ is approximately the mean of $V_{1}$ and $V_{2}$.

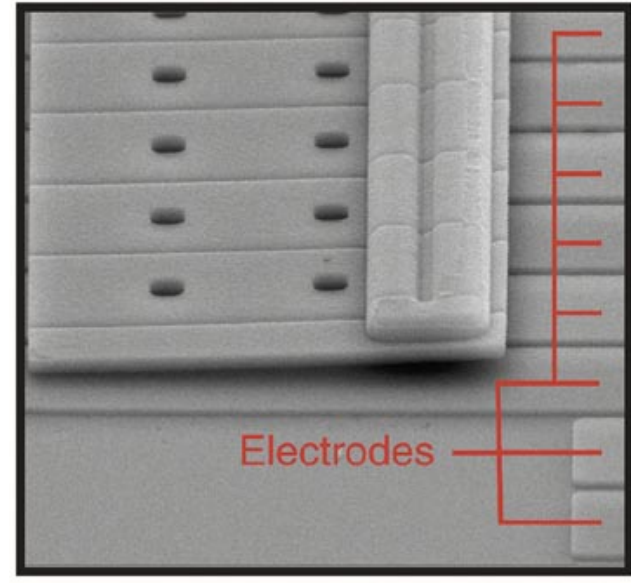

(a)

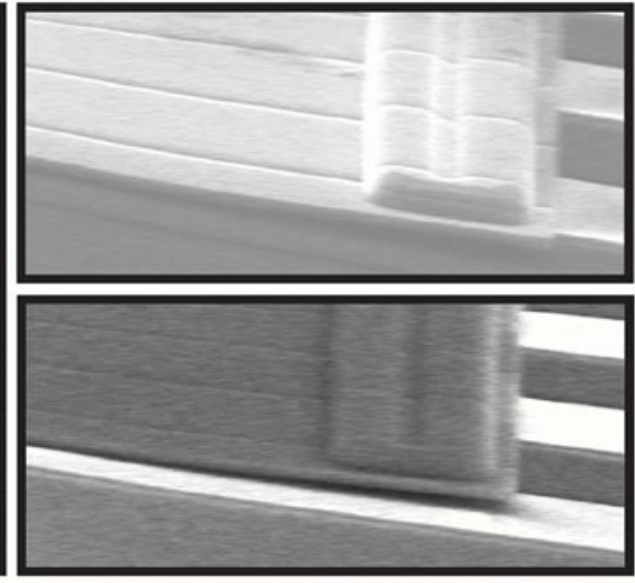

(b)

Fig. 3 (a) Electron micrograph of a capacitively coupled untethered scratch drive actuator atop an array of insulated elctrodes. (b) Two frames extracted from an SEM video of an untethered actuator in motion. At top, a small dc priming voltage can be seen on the electrodes. At bottom, a large voltage between the electrodes pulls the scratch drive plate into flat contact with the substrate. The changes in resolution and intensity between the top and the bottom frames reflect the change in capacitively coupled voltage on the actuator.

The capacitances $C_{1}$ and $C_{2}$ are proportional to the area of overlap between the scratch drive plate and the low-voltage and high-voltage electrodes, respectively. If the dimensions of the actuator greatly exceed the width of each electrode, then the area of high-voltage overlap will always be maintained roughly equal to the area of low-voltage overlap. So, the potential induced between the actuator and any of the electrodes beneath it will be roughly half that applied between the electrodes themselves, regardless of the position and orientation of the drive.

Charging of this circuit produces the electrostatic attraction between the scratch drive and the electrodes, which in turn bows the actuator's plate downward and its bushing forward as shown in Fig. 3.

We have verified this power delivery mechanism by driving untethered scratch drive actuators in a variety of directions, as shown in Fig. 4. In particular, we have successfully driven these actuators in parallel, perpendicular, and diagonal directions rel- ative to the underlying electrodes. In addition, the power delivery mechanism was demonstrated to be robust with respect to the electrode width. Actuators were even observed to continue operating (albeit at reduced speed) after walking off of the main Poly0 electrode panels (18 $\mu \mathrm{m}$ width, $20 \mu \mathrm{m}$ pitch) onto the connecting Poly0 wires ( $6 \mu \mathrm{m}$ width, $20 \mu \mathrm{m}$ pitch) that deliver power to the main electrodes from the bonding pads (see Table V, entries marked $\dagger$ ).

\section{DeVICE Design}

The major components of the untethered actuators are the scratch drive plates, the bushings, and the electrodes. In addition, we must fabricate anchors that provide physical connection of the actuators to the substrate during processing, and sacrificial tethers that connect the actuators to the anchors. Removal of the sacrificial tethers will be described in Section VI. In order 

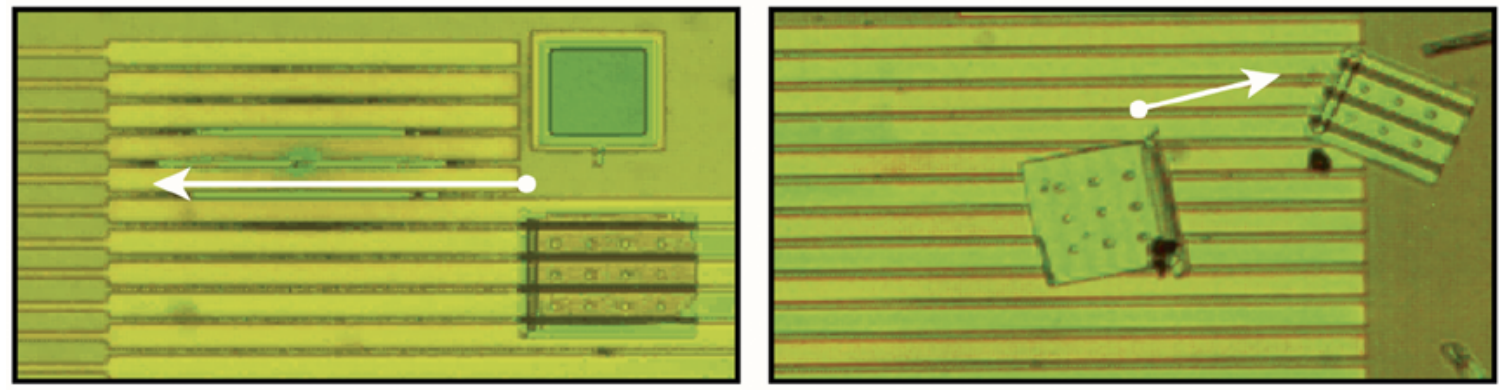

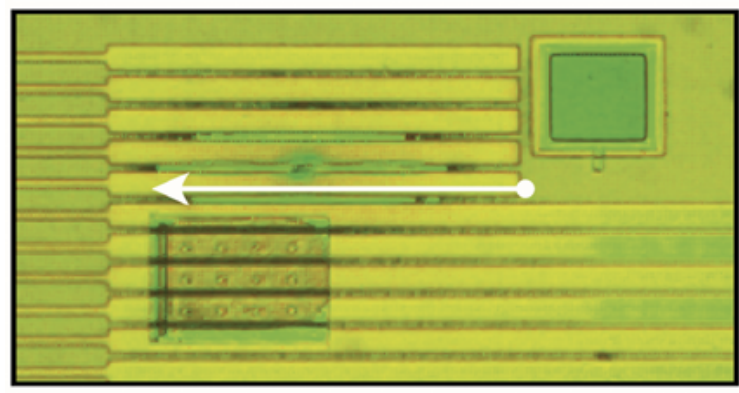

(a)

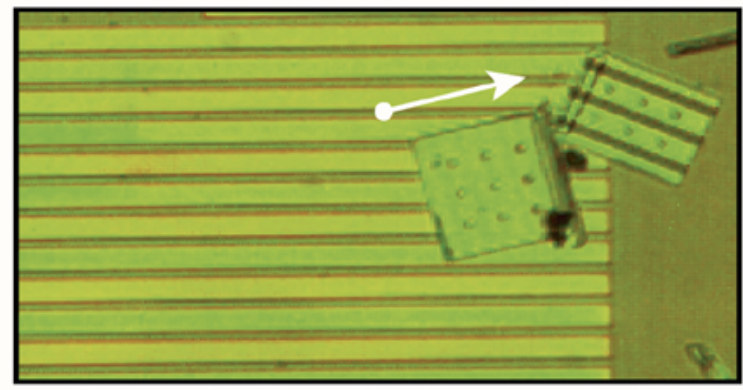

(b)

Fig. 4 Motion of untethered scratch drive actuators is independent of their orientation relative to the underlying electrodes. Here, frames extracted from video of the devices in motion show untethered actuators moving: (a) parallel to the electrodes and (b) diagonally with respect to the electrodes.

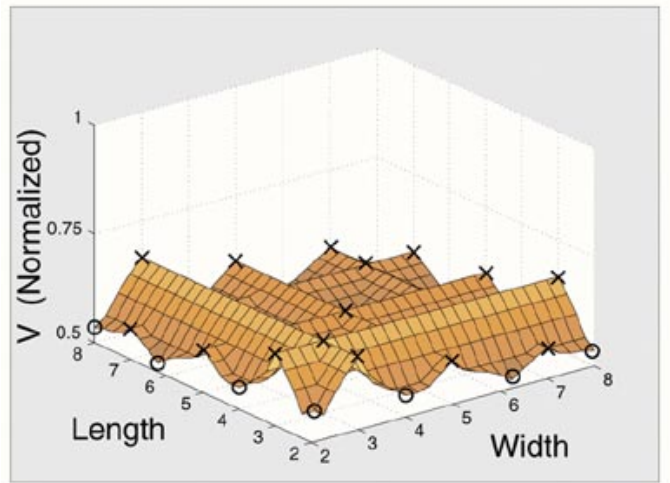

(a)

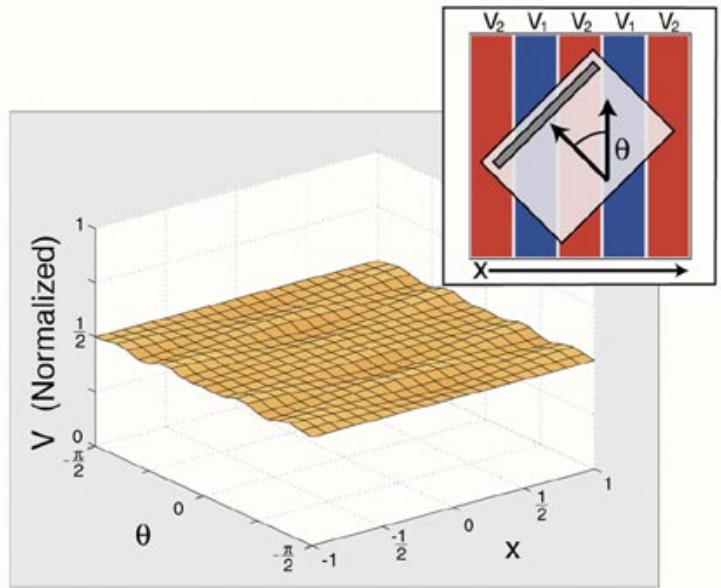

(b)

Fig. 5. Plate design for untethered scratch drive actuators. The size of an untethered scratch drive actuator relative to its underlying electrodes can have significant effect on the voltage delivered to the device. Ideally, this voltage will be independent of the actuator's position and orientation. The length and width of the actuator can be chosen to make this approximately true. Part (a) shows the maximum possible deviation in voltage for an actuator in any position or orientation. The maximum normalized plate voltage $\left(V_{\text {plate }}-V_{1}\right) /\left(V_{2}-V_{1}\right)$ is plotted against the actuator's length and width (expressed as multiples of the electrode width). Maximum deviation from the mean voltage (normalized voltage $=0.5$ ) is small for actuators that are large compared to the electrode width, and for actuators whose sides are even integer multiples of the electrode width. Good locations in the design space are marked here with $\bigcirc$ 's, and locations leading to highly variable power delivery are marked with $\times$ 's. Part (b) shows the normalized plate voltage for one of the actuator designs that we fabricated, as a function of position and orientation defined relative to the electrodes as shown in the inset. This actuator has a length of $120 \mu \mathrm{m}$, a width of $80 \mu \mathrm{m}$, and lies above a series of $20-\mu \mathrm{m}$-wide electrodes. The normalized voltage is approximately 0.5 for all poses, with a maximum deviation of 0.0158 .

to leverage existing MEMS infrastructure, we have fabricated the actuators using the PolyMUMPs multiproject wafer process provided by Cronos Integrated Microsystems [33].

\section{A. Electrode Design}

Electrodes are fabricated from the Poly0 layer, and are designed as long, narrow strips to make it easy to cover a large area without complicated wiring. The electrodes are $18 \mu \mathrm{m}$ wide, with a $2 \mu \mathrm{m}$ interelectrode separation. That is to say, the elec- trode pitch is always maintained at $20 \mu \mathrm{m}$. When wires need to cross in order to provide different signals to adjacent electrodes, electrical bridges are introduced on the Poly1 layer.

\section{B. Scratch Drive Design}

It is important that the voltage delivered to the actuator be approximately independent of the actuator's position and orientation (see Section III). If the electrodes are very thin relative to the dimensions of the actuator, this will always be the case. However, 

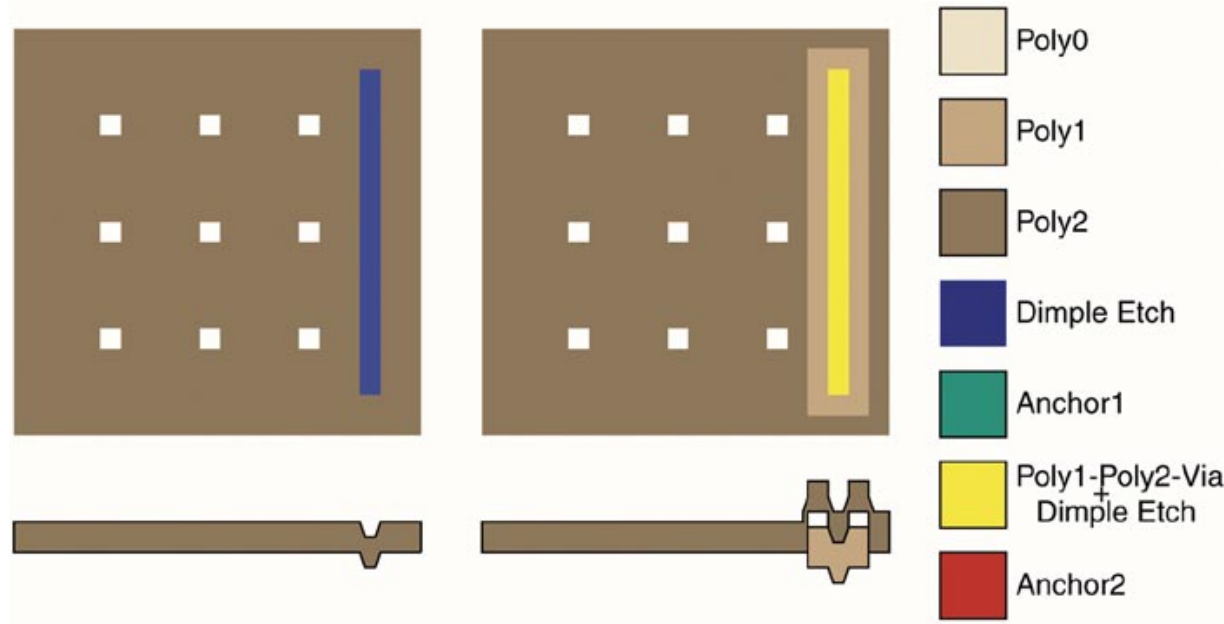

Fig. 6 Layout of the scratch drive actuator. Two types of bushings are shown. (a) Dimple etch is used to form a $0.75 \mu \mathrm{m}$ bushing in a plate form Poly2. (b) Sheet of Poly1 is connected to the Poly2 plate by a thin line of Poly1-Poly2-Via etch. Dimple etch combines with this to form a $1.5 \mu \mathrm{m}$ bushing

since the scratch drive actuators must have plate dimensions on the order of $100 \mu \mathrm{m}$, and since the electrode pitch is $20 \mu \mathrm{m}$, it is possible for the voltage to vary somewhat. The amount that the voltage can vary depends on the dimensions of the scratch drive. The variation in the voltage applied to the actuator can be minimized if the length and width of the scratch drive are both even integer multiples of the electrode pitch, as shown in Fig. 5.

The scratch drive plates are fabricated from a single sheet of Poly2 that is either 80 or $120 \mu \mathrm{m}$ wide, and either $40,60,80$, or $100 \mu \mathrm{m}$ long. This allows the plate dimensions to be (in all but the $60 \mu \mathrm{m}$ case) even integer multiples of the electrode pitch. In this way, the power delivered to the devices can be maintained at a constant level, regardless of their position and orientation. The plates are patterned with holes at $30 \mu \mathrm{m}$ intervals to make the underlying oxide accessible to the etchant during sacrificial release.

Two different scratch drive bushings were designed with heights of $0.75 \mu \mathrm{m}$ and $1.5 \mu \mathrm{m}$. The shorter bushings were defined by conformalities in the Poly2 layer resulting from the Dimple Etch pattern. Taller bushings combined a Dimple Etch conformality with a sheet of Poly1 anchored to the Poly2 layer. In these latter designs, the Polyl-Poly2-Via etch adds an additional $0.75 \mu \mathrm{m}$ to the bushing height. The bushings must be wider than the interelectrode spacing to prevent the actuators from getting stuck in surface features. Since the PolyMUMPs design rules require the spacing between the electrodes to be at least $2 \mu \mathrm{m}$, the bushings were designed with widths of 4,6 , and $8 \mu \mathrm{m}$. Layouts for scratch drives with both bushing heights appear in Fig. 6.

\section{Sacrificial Tether Design}

Substrate anchors are formed from a sheet of Poly2 connected by the Anchor2 etch to an underlying sheet of Poly0. The scratch drive actuators are connected to these anchor points by means of sacrificial tethers. These tethers, shown in Fig. 7, are composed of simple beams on the Poly2 layer. These beams are notched where they join the actuators so that, when under flexure, they concentrate stress at the joint. This way, the tethers break cleanly, and do not leave a significant flange of material on the actuators after release.

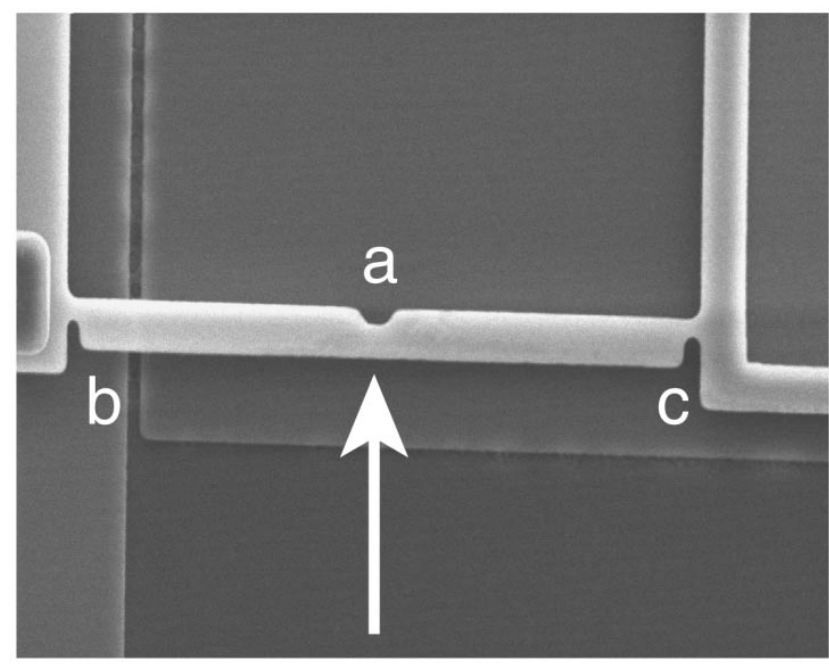

Fig. 7 SEM of a tether used in the manual release process. The tether is creased in the center (a) and scored where it joins the actuator and the anchor (b) and (c), so that a very light lateral pressure with a microprobe will snap it at its endpoints.

\section{Component Integration}

If the actuators are fabricated directly above the electrodes, they will exhibit conformalities corresponding to the interelectrode gaps. For this reason, it is best to fabricate the actuators above a flat region of the die. The actuator must then be moved into position above the electrodes at some point prior to operation. Section VI presents a way that this placement can be performed in an automated fashion.

\section{FABRICATION PROCESS}

Following the sacrificial etch that completes the PolyMUMPs process, there is no layer of insulator between the Poly2 layer (on which we fabricate the actuators) and the Poly0 layer (on which we fabricate the electrodes). For power to be delivered to the devices through the process described in Section IV, we must add an insulating layer between Poly0 and Poly2. In this section, we describe a postprocessing sequence for providing 

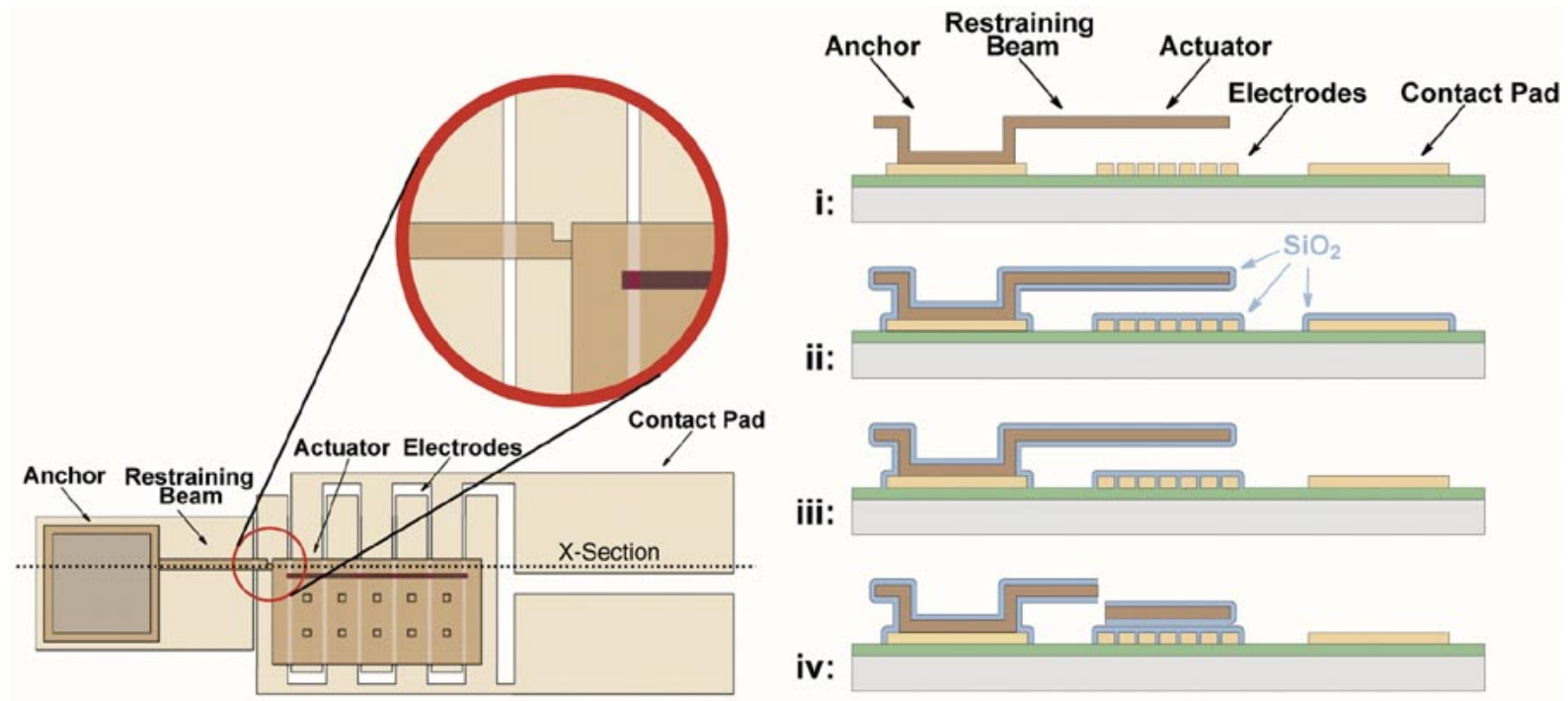

Fig. 8 Plan view and cross-sections of the postprocessing sequence. i: PolyMUMPs sacrificial release produces suspended microstructures. ii: Wet oxidation produces insulator on both the top and bottom of the released devices. iii: Insulator is etched from contact pads to allow power to be delivered to the electrodes. iv: Restraining beams are broken at the score mark (left) to release the actuators.

this intermediate insulating layer on released polysilicon microstructures.

Of course, when devices are received from the PolyMUMPs process, there is already a layer of silicon dioxide between the Poly0 and Poly2 layers-namely, the first and second sacrificial oxides. However, these layers must be removed in order to release the devices. A portion of this oxide must be replaced to provide insulation, but the devices must remain released.

We use wet thermal oxidation to grow a new layer of insulation around the released devices. This leads to a number of important considerations.

First, all released devices must be supported above the surface of the substrate, so as to expose them to the oxidant through the carrier gas, and prevent them from fusing to underlying layers during oxidation. It is very important, therefore, that the effects of stiction following the release process be as small as possible. For this reason, we dry the devices using supercritical $\mathrm{CO}_{2}$.

Second, there must be no gold (or other high-mobility materials) on the die. Gold will diffuse through the nitride layer during oxidation, causing short circuits. For this reason, the PolyMUMPs Metal layer is not used. Electrical wiring is done with the Poly0 layer, and contact pads are fabricated from stacks of all three polysilicon layers.

Third, a means must be provided for making electrical contact with the devices once processing is complete. During oxidation, the contact pads will become insulated, and this insulator must be subsequently removed. We have done this by using photolithography followed by a buffered hydrofluoric acid wet chemical etch. Narrow channels between the released devices and the substrate provide opportunities for residual photoresist scum from this process step to adhere to the die. Care must be taken, as described below, to avoid this problem.

These three considerations lead to the process described in Fig. 8 and Table I. Note the unusually low $\left(550{ }^{\circ} \mathrm{C}\right)$ temperature of the oxidation step. Because the oxidation is performed after sacrificial release, oxide will grow on both the top and the underside of all released devices. However, since the channel between a released device and the substrate is only $2.75 \mu \mathrm{m}$ wide, oxidants are delivered more slowly to the underside of a device than to its top surface. As a result, oxide growth is uneven on the two surfaces. Stress will not be balanced on the top and bottom of the device, resulting in out-of-plane curvature.

A slow oxidation at low temperature resolves this problem. The devices were oxidized at $550{ }^{\circ} \mathrm{C}$ for a duration of 8 days. Oxide thickness data were obtained through ellipsometric measurements of n-type silicon test wafers that shared the furnace with the PolyMUMPs dice. Using this process, the curvature produced in the released structures is negligible. Fig. 8(ii) shows the devices after the oxidation step has been performed.

Following oxidation, the dice undergo photolithography to open contact holes above the pads. The dice are first spin-coated in hexamethyl disilazane (HMDS) to promote adhesion of the photoresist to the oxidized surfaces, and then in photoresist (Shipley 1813, Shipley Company, Marlborough, MA) and exposed. The pattern is then transferred into the oxide with a $10: 1$ buffered hydrofluoric acid wet chemical etch.

After etching contact holes, the photoresist is removed by a 10 -min soak at $60^{\circ} \mathrm{C}$ in an amine-based positive photoresist stripper (ACT, Ashland-ACT, Eaton, PA) using gentle agitation. This removes the top coat of photoresist, and also any resist that has been trapped in the $2.75-\mu \mathrm{m}$ channel between the released devices and the underlying substrate. This is followed by a triple rinse in deionized water to wash away the solvent. The use of hot ACT solvent for photoresist removal at this step reduces stiction difficulties in the completed devices.

The dice are transferred to an ethanol bath, and dried once again in supercritical $\mathrm{CO}_{2}$. To complete the process, the dice are then wire-bonded to ceramic leadless chip carriers. Because the contact pads are bare silicon, wire bonding must be performed at a temperature of $120^{\circ} \mathrm{C}$ to obtain adequate adhesion to the pads. At this point, the chemical processing is complete, and the devices appear as shown in Fig. 8(iii). It remains only to remove 
TABLE I

SEQUENCE of Process Steps Used to PERForm InSERTION OF DiELECTRIC LAYERS

\begin{tabular}{|c|c|}
\hline Process Step & Method \\
\hline PolyMUMPs process: & Performed by Cronos Integrated Microsystems [33]. \\
\hline Sacrificial Release: & $\begin{array}{l}\text { PolyMUMPs release etch. } \\
\mathrm{CO}_{2} \text { critical-point dry. }\end{array}$ \\
\hline Oxide Growth: & $\begin{array}{l}\text { 8-day } 550^{\circ} \mathrm{C} \text { wet thermal oxidation. } \\
\text { Carrier Gas: Nitrogen } \\
\text { Flow Rate: } 1.5 \mathrm{SCFS} \\
\text { Bubbler Temperature: } 94^{\circ} \mathrm{C}\end{array}$ \\
\hline Pattern Contact Holes: & $\begin{array}{l}\text { Spin on HMDS at } 4000 \text { RPM. } \\
\text { Spin on photoresist (Shipley 1813) at } 4000 \text { RPM. } \\
\text { Soft-bake on hot plate for } 3 \text { minutes at } 120^{\circ} \mathrm{C} \text {. } \\
\text { Expose through dark-field mask. } \\
\text { Post-bake on hot plate for } 3 \text { minutes at } 120^{\circ} \mathrm{C} \text {. }\end{array}$ \\
\hline Contact Etch: & $\begin{array}{l}\text { 5-min. 10:1 BHF etch. } \\
\text { DI triple rinse. } \\
\text { 10-min. } 60^{\circ} \mathrm{C} \text { ACT soak. } \\
\text { DI triple rinse. } \\
\mathrm{CO}_{2} \text { critical-point dry. }\end{array}$ \\
\hline
\end{tabular}

the tethers that were used to hold the devices above the substrate throughout the fabrication process. This mechanical release step is described in detail in the following section.

Yield measurements taken from devices produced through this process are as follows. Ninety-four percent of the devices survived the oxidation and lithographic postprocesses without visible damage. Of those, $74 \%$ survived the mechanical release step without fracture. Of those, $24 \%$ were operational, for a cumulative process yield of $17 \%$. Because each untethered actuator is independent of the others, yield difficulties do not have multiplicative effects within a die, as they would, for example, in microarrays [34]-[37]. So, low yields can be compensated by fabricating a larger number of devices than are required.

\section{MECHANICAL RELEASE}

Following the postprocessing sequence described in Section $\mathrm{V}$, the actuators are still attached to the substrate by physical tethers. These tethers, shown in Figs. 2 and 7, are strong enough to withstand the rigors of the fabrication process, but are designed to break in a controlled fashion under a small amount of mechanical pressure.

We have performed controlled mechanical release of the actuators in two ways. First, we have released the devices manually, with a pair of microprobes. This is simple to do, and is quite practical for research purposes, where the number of actuators to be released is small. In a production setting, where the number of actuators would be much larger, an automated solution would be required. Therefore, we have developed a means by which the actuators can perform their own electromechanical release, requiring only an electrical signal from the operator.

\section{A. Manual Release}

We have released the devices manually, with a pair of microprobes. The first probe applies downward pressure on the scratch drive plate, creating a large area of contact with the substrate and immobilizing the actuator. The second microprobe applies lateral pressure on the tether. The stress from this action is concentrated in the score mark that was fabricated in the tether, resulting in fracture at the desired location. The lateral force required to break the tether can be reduced by scoring the beam in three locations as shown in Fig. 7. This reduces the downward pressure required to immobilize the actuator, resulting in a lower likelihood of damage to the device.

Once the actuator has been released, it must be positioned above a sheet of electrodes before operating. ${ }^{1}$ This process is performed manually with a microprobe. Since the power delivery mechanism is independent of position and orientation, very little precision is required in this placement step. Once the actuators have been mechanically released and placed on top of the electrodes, they are fully operable.

\section{B. Automated Self-Release}

For purposes of batch fabrication, we would like to be able to perform this assembly process in an entirely automated fashion. The process has two steps. The actuator is operated to walk

\footnotetext{
${ }^{1}$ If the actuator were fabricated directly above the electrodes, it would exhibit conformalities above the interelectrode gaps.
} 


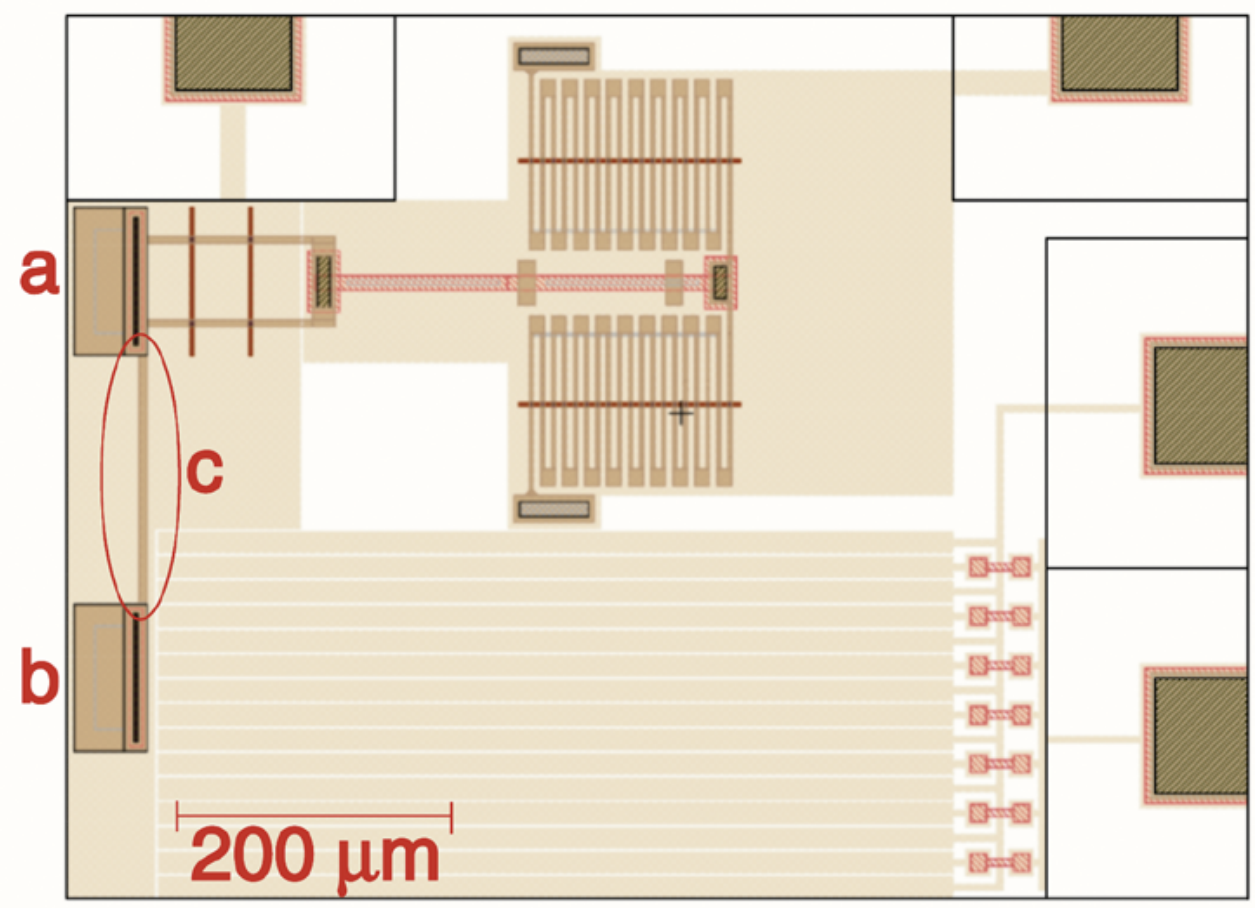

Fig. 9 Layout of the self-release mechanism. The assisting spring-tethered scratch drive (a) moves forward while the target actuator release operation (b) holds down to the substrate by electrostatic attraction. The restraining beam (c) breaks at the score mark (see Fig. 8 adjacent to the untethered actuator).

onto an electrode-covered area of the substrate, and is then electromechanically released from its fabrication tethers. The setup for this assembly process is shown in Fig. 9.

We used a spring-tethered assisting scratch drive actuator to pull the target actuator into position above a set of electrodes. The two actuators are attached at the bushing by a polysilicon beam, which provides both physical and electrical connection. A voltage is applied to both actuators through the spring that anchors the assisting actuator to the nitride-coated substrate.

Initially, both actuators lie over a flat sheet of (oxide-insulated) Poly0, which is connected to ground. A periodic voltage is applied between the actuators and the underlying Poly0 sheet, in order to cause forward motion of both scratch drives. Since the two scratch drives have the same length, width, and bushing height, they move forward at the same speed.

As the actuators move forward, the path of the assisting actuator continues to be paved with a single flat sheet of oxide-insulated Poly0. The path of the target actuator, on the other hand, quickly changes into a sheet of electrode panels. These electrodes are all held at ground potential, so that the motion of both scratch drives continues as before. However, once the target actuator has moved completely onto the electrode surface, a dc voltage is applied to the electrode panels. In this way, as the potential of the scratch drives oscillates between high-voltage and ground, there is always an attractive force between the target actuator and the surface beneath it. So, the target actuator holds down electrostatically to the substrate, thereby causing friction, which immobilizes the actuator with respect to both translation and rotation. The assisting actuator continues moving as before.

As the assisting actuator continues to move, it bends the beam that connects the two actuators. The resulting stress is concentrated in the score mark that was fabricated where the beam joins the target actuator, and the beam breaks at the desired location. Frames captured from video footage of this self-release process are shown in Fig. 10.

At this point, the assembly of the target actuator is complete. A periodic potential applied between the underlying electrodes results in untethered actuation.

\section{DeVICE PeRformance}

Untethered scratch drive actuators are operable over a wide range of design and control parameter values. We operated untethered scratch drives that were 80-120 $\mu \mathrm{m}$ wide, between $40 \mu \mathrm{m}$ and $100 \mu \mathrm{m}$ long, and had bushing heights of $0.75 \mu \mathrm{m}$ and $1.5 \mu \mathrm{m}$. Untethered actuators from two different PolyMUMPs runs were observed to have correct operation. The devices were operated in a variety of directions, including parallel, perpendicular and diagonal relative to the underlying electrodes, and were found to be operable in all orientations.

To operate the devices, half of the electrodes were set to ground potential, while the other half received a square-wave signal. We measured the speed of actuators with 80 - and $100-\mu \mathrm{m}$ plate dimensions and $0.75-\mu \mathrm{m}$ bushings. Many different drive signals were used, as shown in Tables II and III. These signals incorporated peak-to-peak electrode voltages ${ }^{2}$ of $100 \mathrm{~V}$ or $120 \mathrm{~V}$, and frequencies of $50 \mathrm{~Hz}, 100 \mathrm{~Hz}, 1 \mathrm{kHz}$, or $10 \mathrm{kHz}$. (During these experiments, the underlying substrate was held at ground potential.) The motion of the devices was filmed, ${ }^{3}$ and the video frames were extracted as still images. Interframe motion esti-

\footnotetext{
${ }^{2} \mathrm{As}$ discussed in Section III, interelectrode voltages of $100 \mathrm{~V}$ and $120 \mathrm{~V}$ result in $50 \mathrm{~V}$ and $60 \mathrm{~V}$ potentials between the actuator and the electrodes, respectively.

${ }^{3}$ Video was taken with both analog and digital cameras, and was then imported for analysis and processing with Adobe Premiere and Matlab.
} 


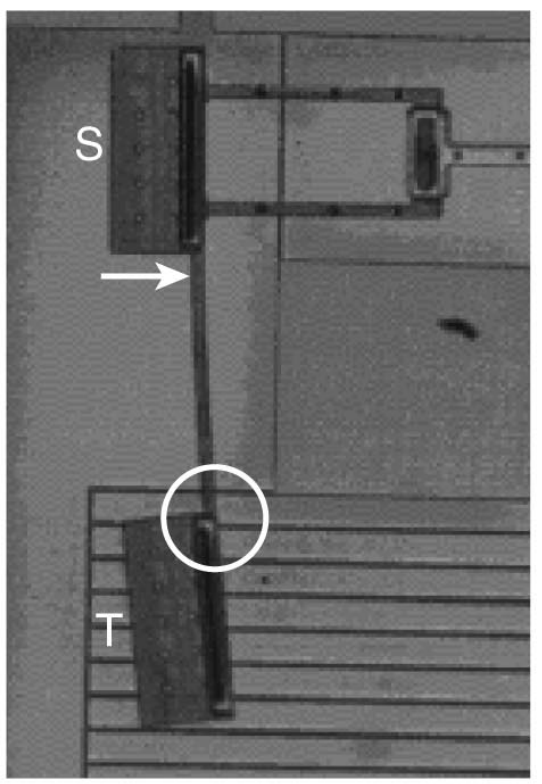

(a)

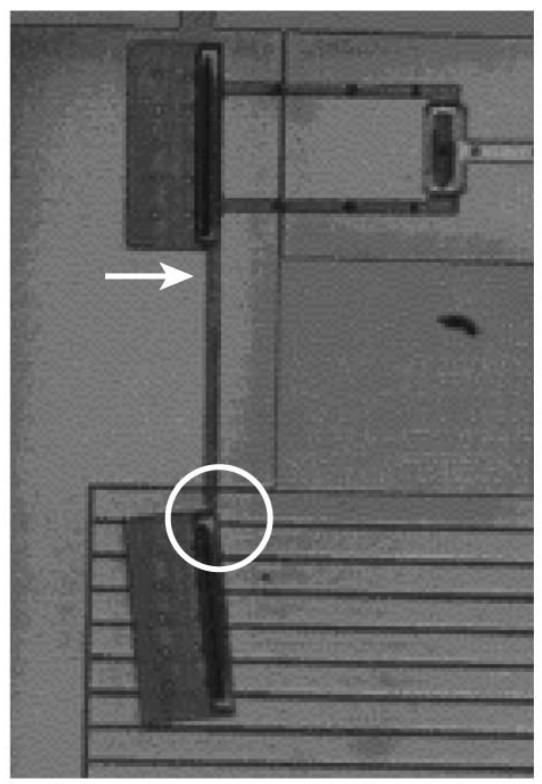

(b)

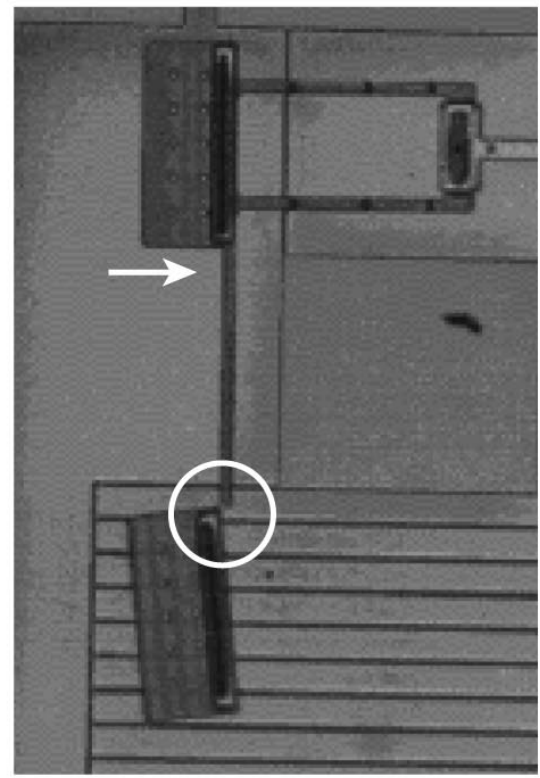

(c)

Fig. 10 Frames captured from video footage of the self-release process. (a) Target drive (T) holds down electrostatically to the substrate. (b) Assisting drive (S) continues actuating. (c) Tether snaps at the score mark, releasing the target actuator

TABLE II

AVERAGE SPEED AS A FUNCTION OF FREQUENCY $($ Plate Voltage $=50 \mathrm{~V})$

\begin{tabular}{|c|c|c|c|c|c|c|}
\hline \multirow[t]{2}{*}{ Device } & \multirow{2}{*}{$\begin{array}{c}\text { Length } \\
\qquad(\mu m)\end{array}$} & \multirow{2}{*}{$\begin{array}{c}\text { Width } \\
(\mu m)\end{array}$} & \multicolumn{4}{|c|}{ Speed $(\mu \mathrm{m} / \mathrm{sec})$} \\
\hline & & & $50 \mathrm{~Hz}$ & $100 \mathrm{~Hz}$ & $1 \mathbf{k H z}$ & $10 \mathrm{kHz}$ \\
\hline 1 & 80 & 100 & & 6.0 & 33 & \\
\hline 2 & 80 & 100 & & 4.6 & 25 & \\
\hline 3 & 100 & 80 & & 5.1 & 34 & \\
\hline 4 & 100 & 80 & & & 38 & \\
\hline 5 & 100 & 80 & 1.8 & 4.0 & 37 & \\
\hline 6 & 100 & 80 & 1.7 & 3.7 & 39 & \\
\hline 7 & 100 & 80 & 2.4 & 4.8 & 38 & \\
\hline 8 & 100 & 80 & & & 32 & 262 \\
\hline 9 & 100 & 80 & 2.5 & & 28 & 279 \\
\hline 10 & 100 & 80 & 3.1 & & 40 & \\
\hline 11 & 100 & 100 & & & 47 & \\
\hline Mean & & & 2.3 & 4.7 & 35.5 & 270.5 \\
\hline Std. Dev. & & & 0.6 & 0.8 & 6.1 & 12.0 \\
\hline $\begin{array}{l}\text { Predicted Range } \\
\text { (Eqs. 2-3) }\end{array}$ & & & $1.4-1.7$ & $2.8-3.4$ & $28-33$ & $280-330$ \\
\hline
\end{tabular}

mates were then used to determine the speed of the actuators based on the frame rate of the video. The resulting data are shown in Tables II and III, and Fig. 11. The average step size of the actuators, $\Delta x$, can be calculated from the ratio of the actuator speed to the frequency of the waveform.

At frequencies near $100 \mathrm{kHz}$, the motion of the actuators occurs in short, sporadic bursts on the order of a tenth of a second.
So, it is more appropriate to measure the maximum speed between any two adjacent frames of the video than to take an average speed across many frames. Data taken in this fashion for actuators driven at $10 \mathrm{kHz}$ and $100 \mathrm{kHz}$ appear in Tables IV and $\mathrm{V}$, and in Fig. 11.

From the measured speed of an actuator and the frequency of the applied waveform, we can calculate the average step size 
TABLE III

AVERAGE SPEED AS A FUNCTION OF FREQUENCY

$($ Plate Voltage $=60 \mathrm{~V})$

\begin{tabular}{|c|c|c|c|c|c|}
\hline \multirow[t]{2}{*}{ Device } & \multirow{2}{*}{$\begin{array}{c}\text { Length } \\
(\mu \mathrm{m})\end{array}$} & \multirow{2}{*}{$\begin{array}{c}\text { Width } \\
(\mu m)\end{array}$} & \multicolumn{3}{|c|}{ Speed $(\mu \mathrm{m} / \mathrm{sec})$} \\
\hline & & & $100 \mathrm{~Hz}$ & $1 \mathrm{kHz}$ & $10 \mathrm{kHz}$ \\
\hline 12 & 100 & 60 & 2.5 & 22 & 183 \\
\hline 13 & 100 & 80 & 3.6 & 34 & 316 \\
\hline 14 & 100 & 80 & 2.5 & & \\
\hline 15 & 100 & 80 & 2.7 & 28 & 285 \\
\hline 16 & 100 & 80 & 4.1 & 27 & 265 \\
\hline 17 & 100 & 80 & 3.7 & 37 & 313 \\
\hline 18 & 100 & 80 & 3.1 & 31 & \\
\hline Mean & & & 3.2 & 29.8 & 272.4 \\
\hline Std. Dev. & & & 0.6 & 5.3 & 54.2 \\
\hline $\begin{array}{l}\text { Predicted Range } \\
\text { (Eqs. 2-3) }\end{array}$ & & & $3.1-3.7$ & $31-37$ & $310-370$ \\
\hline
\end{tabular}

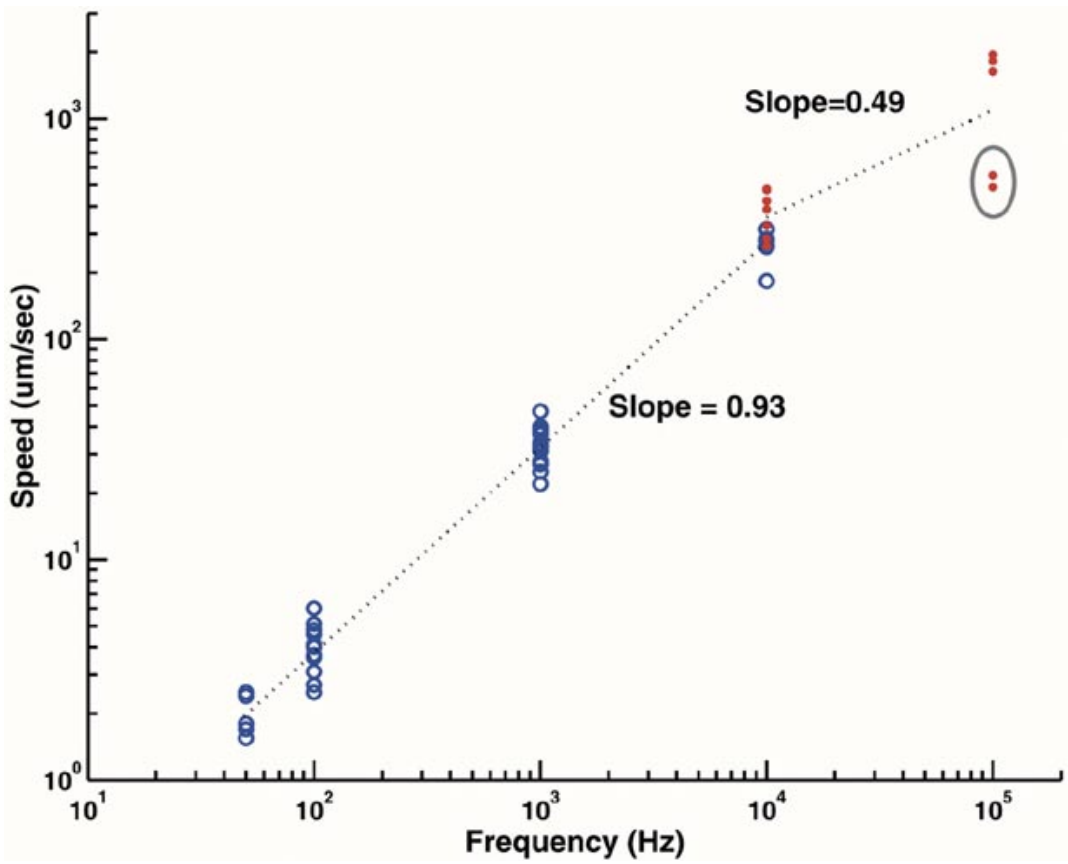

Fig. 11 Actuator speed as a function of frequency. Control of untethered microactuators is possible at a wide range of frequencies and speeds. Data points marked with "o" (in blue) reflect average speeds. Data points marked with a dot (in red) reflect maximum interframe speeds. Circled data points (in gray) reflect motion that occurred off of the intended electrodes and above (much thinner) electrical wiring. The slope of the plot shown in log-log space above, reflects a slight decrease in the step size of the actuators as the frequency increases across four orders of magnitude.

during travel. A scratch drive actuator's step size can be modeled analytically as follows [29]:

$$
\Delta x=P \sqrt{V}
$$

where $P$ is a constant defined by

$$
P=\left(\frac{27 k \epsilon_{0} h^{6}}{4 E d t^{3}}\right)^{\frac{1}{4}}
$$

in which $k$ is the dielectric constant, $\epsilon_{0}$ is the permittivity of free space, $h$ is the bushing height, $E$ is the Young's modulus of the plate material, $d$ is the thickness of the insulating layer, and $t$ is the thickness of the scratch drive plate.

To calculate the expected step size for the actuators used in this experiment, we need to know the thickness of the insulating oxide layer $(d)$. While ellipsometric measurements of test wafers give us an approximation of the oxide thickness on the top of the electrodes (500 $\AA$ ), the oxide on the underside of the 
TABLE IV

MaXimum SPEED FOR High-FreQueNCy DRIVE Signals $($ Plate Voltage $=50 \mathrm{~V})$

\begin{tabular}{c|c|c|c|c}
\hline Device & Length & Width & \multicolumn{2}{|c}{ Speed $(\mu \mathrm{m} / \mathrm{sec})$} \\
& $(\mu \mathrm{m})$ & $(\mu \mathrm{m})$ & $\mathbf{1 0} \mathbf{~ k H z}$ & $\mathbf{1 0 0} \mathbf{~ k H z}$ \\
\hline 8 & 100 & 80 & 327 & \\
9 & 100 & 80 & 423 & \\
10 & 100 & 80 & & 1635 \\
\hline Mean & & & $\mathbf{3 7 5 . 0}$ & $\mathbf{1 6 3 5}$ \\
$\begin{array}{c}\text { Std. Dev. } \\
\text { Predicted Range } \\
\text { (Eqs. 2-3) }\end{array}$ & & & $\mathbf{6 7 . 9}$ & $\mathbf{N} / \mathbf{A}$ \\
\hline
\end{tabular}

TABLE $\mathrm{V}$

MAXIMUM SPEED For High-FreQueNCy DRIVE Signals $($ Plate Voltage $=60 \mathrm{~V})$

\begin{tabular}{c|c|c|c|c}
\hline Device & Length & Width & \multicolumn{2}{|c}{ Speed $(\mu \mathrm{m} / \mathrm{sec})$} \\
& $(\mu \mathrm{m})$ & $(\mu \mathrm{m})$ & $\mathbf{1 0} \mathbf{~ k H z}$ & $\mathbf{1 0 0} \mathbf{~ k H z}$ \\
\hline 12 & 100 & 60 & 264 & \\
13 & 100 & 80 & 286 & \\
19 & 100 & 80 & & 1825 \\
14 & 100 & 80 & 387 & $488^{\dagger}$ \\
15 & 100 & 80 & 386 & $553^{\dagger}$ \\
16 & 100 & 80 & 479 & \\
17 & 100 & 80 & 474 & 1947 \\
\hline Mean & & & $\mathbf{3 7 9 . 3}$ & $\mathbf{1 8 8 6}$ \\
Std. Dev. & & & $\mathbf{9 0 . 6}$ & $\mathbf{8 6 . 3}$ \\
$\begin{array}{c}\text { Predicted Range } \\
\text { (Eqs. 2-3) }\end{array}$ & & $\mathbf{3 1 0}-\mathbf{3 7 0}$ & $\mathbf{3 1 0 0}-\mathbf{3 7 0 0}$ \\
\hline
\end{tabular}

actuators grows at a slower rate, due to restricted gas transport through the small channel between the actuator and the substrate. Since the total insulator thickness, $d$, is the the sum of these two layers, we modeled the thickness to lie between 500 and $1000 \AA$. For actuators driven at $50 \mathrm{~V}$, this predicts a step size between 28 and $33 \mathrm{~nm}$. For actuators driven at $60 \mathrm{~V}$, the range is from 31 to $37 \mathrm{~nm}$. The observed values fell near this predicted range, and are shown in Tables II and IV. ${ }^{4}$

The actuators achieved speeds of over $300 \mu \mathrm{m} / \mathrm{s}$ at drive frequencies of $10 \mathrm{kHz}$. At higher frequencies, even higher speeds were observed. At $100 \mathrm{kHz}$, the maximum drive frequency allowed by the voltage source, actuators were observed to exceed speeds of $1.5 \mathrm{~mm} / \mathrm{s}$ (see Tables IV and V). Occasionally, the devices stopped moving due to electrostatic charge accumulation on the insulating layer. Reversal of the polarity of the drive waveform allowed the devices to continue walking after this oc-

${ }^{4}$ While the average speed of the devices run at $50 \mathrm{~V}$ exceeds that of the devices run at $60 \mathrm{~V}$, this difference is not statistically significant (Student's $t$-test $p<0.01)$. The relationship between drive voltage and step size is discussed extensively in [26], [29]-[31]. curred. Speed measurements were taken during intervals of constant drive waveform polarity.

Video of these untethered actuators can be viewed on the internet at: www.cs.dartmouth.edu/ brd/Research/MEMS/ Movies/

\section{CONCLUSIONS AND FUTURE WORK}

This work provides basic components that enable untethered locomotion at the MEMS scale. We provide a power delivery mechanism that is independent of device location and orientation, an automated release process, and a microfabrication process for building untethered devices. We have demonstrated the feasibility of these components through the development of untethered scratch drive actuators, and have characterized the performance of these devices.

In the course of producing untethered scratch drive actuators, we have developed a novel postprocessing technique for post hoc insertion of dielectric layers between previously-deposited polysilicon films. We have successfully employed this technique to insulate devices fabricated with the PolyMUMPs process. As discussed in Section $\mathrm{V}$, device yield from this process has been measured at $17 \%$. We expect that much of the device loss is due to a combination of stress gradients from oxidation, dielectric breakdown of the oxide, and residual photoresist from the postrelease lithography step. If higher yields are required, lower oxidation temperatures (and longer oxidation times) may be useful to reduce any remaining stress. More aggressive photoresist removal steps, such as oxygen plasma cleaning, may prove beneficial for clearing the undersides of the actuators from residual photoresist. Dielectric breakdown in the oxide was associated with discolorations that suggested contamination prior to oxidation. Cleaner handling practices would likely reduce these defects. In addition, optimization of the device geometry may produce a further increase in yield, as discussed by [27] and [30].

It is hoped that the techniques presented in this paper will be valuable for future work on autonomous locomotion of MEMS devices. Given the ability to locomote freely on the substrate, the natural next step is to devise steering mechanisms and control systems for untethered MEMS actuators. Work is underway to pursue this goal.

\section{ACKNOWLEDGMENT}

Devices were fabricated in part through the PoIyMUMPs process at Crones Integrated Microsystems, and were later wire-bonded at the Micro-Technology Lab of the Massachusetts Institute of Technology. The motion estimation code for measuring actuator speed was generously provided by H. Farid and S. Periaswamy. The authors would like to thank C. Sullivan for the use of bench space in his lab; L. Ray for the use of her Piezo Amplifier; and C. Daghlian and the Rippel Electron Microscopy Lab for the use of their $\mathrm{CO}_{2}$ critical-point drying system, their SEM, and their many helpful suggestions. Finally, many thanks to K. Bohringer, Z. Butler, I. Paprotny, and K. Kotay for their advice and discussions. 


\section{REFERENCES}

[1] S. Baglio, S. Castorina, L. Fortuna, and N. Savalli, "Development of autonomous, mobile micro-electro-mechanical devices," in IEEE International Symposium on Circuits and Systems Proceedings, vol. IV, 2002, pp. $285-288$.

[2] _ "Technologies and architectures for autonomous "MEMS" microrobots," in IEEE International Symposium on Circuits and Systems. Proceedings, vol. II, 2002, pp. 584-587.

[3] T. Ebefors, J. U. Mattsson, E. Kälvesten, and G. Stemme, "A walking silicon micro-robot," Proc. Transducers'99, pp. 1202-1205, June 1999.

[4] P. E. Kladitis and V. M. Bright, "Prototype microrobots for micro-positioning and micro-unmanned vehicles," Sens. Actuators A, Phys., vol. A80, no. 2, pp. 132-137, March 2000.

[5] R. J. Linderman and V. M. Bright, "Optimized scratch drive actuator for tethered nanometer positioning of chip-sized components," Proc. Transducers'2000, pp. 214-217, June 2000.

[6] M. H. Mohebbi, M. L. Terry, K. F. Bohringer, G. T. A. Kovacs, and J. W. Suh, "Omnidirectional walking microrobot realized by thermal microactuator arrays," in Proc. ASME International Mechanical Engineering Congress and Exposition, Nov. 2001, pp. 1-7.

[7] K. Suzuki, I. Shimoyama, and H. Miura, "Insect-model based microrobot with elastic hinges," J. Microelectromech. Syst., vol. 3, no. 1, pp. 4-9, Mar. 1994.

[8] T. Yasuda, I. Shimoyama, and H. Miura, "Microrobot actuated by a vibration energy field," Sens. Actuators A, Phys., vol. 43, pp. 366-370, 1994.

[9] C. Cassier, A. Ferreira, and S. Hirai, "Combination of vision servoing techniques and vr-based simulation for semi-autonomous microassembly workstation," in Proc. IEEE International Conference on Robotics and Automation, May 2002, pp. 1501-1506.

[10] P. Dario, R. Valeggi, M. C. Carrozza, M. C. Montesi, and M. Cocco, "Microactuators for microrobots: A critical survey," J. Micromech. Microeng., p. 141, Sept. 1992

[11] S. Fatikow, J. Seyfried, S. Fahlbusch, A. Buerkle, and F. Schmoeckel, "A flexible microrobot-based microassembly station," in Proc. IEEE International Conference on Emerging Technologies and Factory Automation, 1999, pp. 397-406.

[12] J. Seyfried, S. Fatikow, S. Fahlbusch, A. Buerkle, and F. Schmowkel, "Manipulating in the micro world: mobile micro robots and their applications," in Proc. 31st International Symposium on Robotics, May 1999, pp. 3-9.

[13] H. Worn, F. Schmoeckel, A. Buerkle, J. Samitier, M. Puig-Vidal, S. Johansson, U. Simu, J.-U. Meyer, and M. Bichl, "From decimeter- to centimeter-sized mobile microrobots-the development of the miniman system," in Proc. SPIE Microrobotics and Microassembly III, vol. 4568, 2001, pp. 175-186.

[14] M. Mason, Mechanics of Robotic Manipulation: MIT Press, 2001.

[15] B. R. Donald, J. Jennings, and D. Rus, "Information invariants for distributed manipulation," Int. J. Robot. Res., vol. 16, no. 5, pp. 673-702, 1997.

[16] D. H. Gracias, I. Choi, M. Weck, and G. M. Whitesides, "Meso-scale self assembly," in Algorithmic and Computational Robotics, B. R. Donald, K. Lynch, and D. Rus, Eds. Boston, MA: A. K. Peters, 2000, pp. 1-8.

[17] Z. Butler, R. Fitch, and D. Rus, "Distributed control for unit-compressible robots: goal-recognition, locomotion, and splitting," IEEE/ASME Trans. Mechatron., vol. 7, p. 418, 2002.

[18] Z. Butler and D. Rus, "Distributed motion planning for 3-d unit-compressible robots," Int. J. Robot. Res., Sept. 2003.

[19] K. Kotay and D. Rus, "Locomotion versatility through self-reconfiguration," Robot. Auton. Syst., vol. 26, p. 217, 1999.

[20] D. Rus, "Self-reconfiguring robots," IEEE Intell. Syst., vol. 13, p. 2, 1998.

[21] D. Rus and M. Vona, "Crystalline robots: self-reconfiguration with compressible unit modules," Auton. Robots, vol. 10, 2001.

[22] D. Rus, Z. Butler, K. Kotay, and M. Vona, "Self-reconfiguring robots," Commun. ACM, vol. 45, p. 39, 2002

[23] B. R. Donald, MEMS for Infosecurity, The Office for Domestic Preparedness, Department of Homeland Security, USA, 2000-2003.

[24] B. R. Donald, C. McGray, and D. Rus, "MEMS for Infosecurity," Dartmouth Computer Science, Tech. Rep. TR2003-469, Aug. 2003.

[25] P. Basset, A. Kaiser, P. Bigotte, D. Collard, and L. Buchaillot, "A large stepwise motion electrostatic actuator for a wireless microrobot," in Proc. IEEE International Conference on Micro Electro Mechanical Systems, 2002, pp. 606-609.
[26] T. Akiyama and K. Shono, "Controlled stepwise motion in polysilicon microstructures," J. Microelectromech. Syst., vol. 2, pp. 106-110, Sept. 1993.

[27] T. Akiyama, D. Collard, and H. Fujita, "Scratch drive actuator with mechanical links for self-assembly of three-dimensional MEMS," J. Microelectromech. Syst., vol. 6, pp. 10-17, Mar. 1997.

[28] N. Finch, J. Marchetti, H. Fujita, and J. Guoy, "CAD modeling of scratch drive actuation," in Proc. SPIE. Design, Modeling, and Simulation in Microelectronics, vol. 2639, 2000, pp. 83-89.

[29] K. Hayakawa, A. Torii, and A. Ueda, "An analysis of the elastic deformation of an electrostatic microactuator," Trans. Inst. Elec. Eng. Jpn., Part E, vol. 118-E, no. 3, pp. 205-211, Mar. 1998.

[30] P. Langlet, D. Collard, T. Akiyama, and H. Fujita, "A quantitative analysis of scratch drive actuation for integrated x/y motion system," Proc. Transducers'97, pp. 773-776, June 1997.

[31] L. Li, J. G. Brown, and D. Uttamchandani, "Detailed study of scratch drive actuator characteristics using high-speed imaging," in Proc. SPIE Reliability, Testing, and Characterization of MEMS/MOEMS, vol. 4558, 2001, pp. 117-123.

[32] L. G. Frechette, S. F. Nagle, R. Ghodssi, S. D. Umans, M. A. Schmidt, and J. H. Lang, "An electrostatic induction micromotor supported on gas-lubricated bearings," in Proc. 14th IEEE International Conference on Micro Electro Mechanical Systems, 2001, pp. 290-293.

[33] K. W. Markus, D. A. Koester, A. Cowen, R. Mahadevan, V. R. Dhuler, D. Roberson, and L. Smith, "MEMS infrastructure: the multi-user MEMS processes (MUMPS)," in Proc. SPIE-The International Society for Optical Engineering, Micrormachining and Microfabrication Process Technology, vol. 2639, 1995, pp. 54-63.

[34] J. W. Suh, R. B. Darling, K. F. Bohringer, B. R. Donald, H. Baltes, and G. T. Kovacs, "Fully programmable MEMS ciliary actuator arrays for micromanipulation tasks," in Proc. 2000 IEEE International Conference on Robotics and Automation, 2000.

[35] K. F. Bohringer, J. W. Suh, B. R. Donald, and G. T. Kovacs, "Vector fields for task-level distributed manipulation: experiments with organic micro actuator arrays," in Proc. 1997 IEEE International Conference on Robotics and Automation, 1997.

[36] K. F. Bohringer, B. R. Donald, and N. C. MacDonald, "Single-crystal silicon actuator arrays for micro manipulation tasks," in Proc. 1996 IEEE International Workshop on Micro Electromechanical Systems, 1996.

[37] K. F. Bohringer, B. R. Donald, R. Mihailovich, and N. C. MacDonald, "Sensorless manipulation using massively parallel microfabricated actuator arrays," in Proc. 1994 IEEE International Conference on Robotics and Automation, 1994

[38] T. Lozano-Perez and M. A. Wesley, "An algorithm for planning collision-free paths among polyhedral obstacles," Commun. ACM, vol. 22, no. 10 , pp. $560-570,1979$

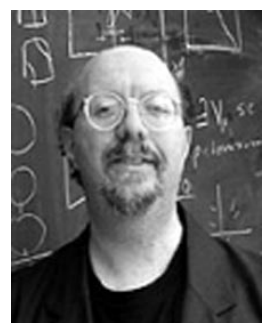

Bruce Randall Donald (S'85-M'87) received the B.A. degree from Yale University, New Haven, CT, in 1980, the S.M. degree in electrical engineering and computer science from Massachusetts Institute of Technology (MIT), Cambridge in 1984, and the Ph.D. degree from MIT in 1987.

He is the Joan P. and Edward J. Foley Professor in the Computer Science Department at Dartmouth College, Hanover, $\mathrm{NH}$, where he holds a joint appointment in the Department of Chemistry and the Department of Biological Sciences. From 1987 to 1998, he was a Professor in the Computer Science Department at Cornell University, Ithaca, NY, with a joint appointment in applied mathematics. He has worked in research, visiting, and faculty positions at Harvard University, Cambridge, Stanford University, Stanford, CA, Interval Research Corporation, and MIT. He has been a National Science Foundation Presidential Young Investigator. He has worked in several research areas, including robotics, microelectromechanical Systems (MEMS), computational biology, graphics, and geometric algorithms.

Dr. Donald was awarded a Guggenheim Fellowship for his work on algorithms for structural proteomics in 2001. Research in the Donald laboratory is funded by NSF and NIH. 


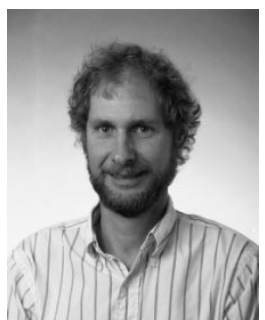

Christopher G. Levey (A'93) received the B.A. degree in physics from Carleton College, Northfield, MN, in 1977 and the Ph.D. degree in physics (electrical engineering minor) from the University of Wisconsin-Madison in 1984

He was then a Member of the Technical Staff at AT\&T Bell Labs until 1986, when he joined the faculty of Dartmouth College, Hanover, NH. His research as a faculty member in the Physics Department involved optical, thermal, and field effect properties of high temperature superconductors. After a sabbatical leave at Bellcore, he joined the Thayer School of Engineering at Dartmouth College in 1993. His research there has included work on a variety of microfabricated devices, including binary optics, chemical (smell) actuators, integrated inductors, and walking MEMS actuators. He is the Director of the Microengineering Laboratory at the Thayer School of Engineering at Dartmouth College.

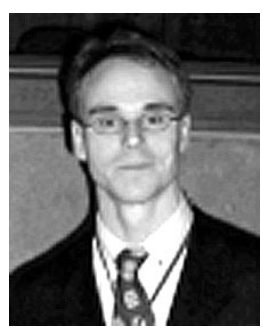

Craig D. McGray (S'02) received the B.A. degree from Middlebury College, Middlebury, VT, in 1995. $\mathrm{He}$ is currently working toward the Ph.D. degree at Dartmouth College, Hanover, NH, where he works in the Dartmouth Robotics Laboratory and the Microengineering Laboratory at the Thayer School of Engineering.

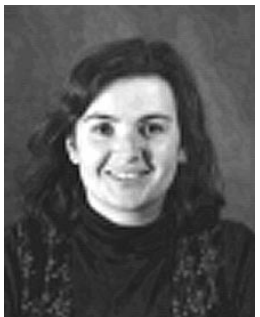

Daniela Rus (M'02) received the Ph.D. degree in computer science form Cornell University, Ithaca, NY.

She is Professor of Computer Science at Dartmouth College, Hanover, NH, where she founded and directs the Dartmouth Robotics Laboratory. She also cofounded and codirects the Transportable Agents Laboratory and the Dartmouth Center for Mobile Computing. Her research interests include distributed robotics, self-reconfiguring robotics, mobile computing, and information organization.

Dr. Rus was the recipient of an NSF Career award, and an Alfred P. Sloan Foundation Fellowship. She is a class of 2002 MacArthur Fellow.

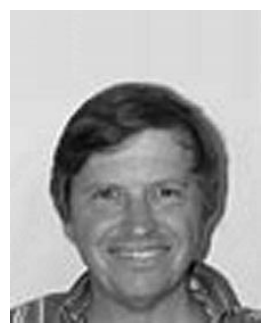

Mike Sinclair received the B.S. and M.S. degrees in electrical engineering from Georgia Institute of Technology (Georgia Tech), Atlanta.

$\mathrm{He}$ is a Senior Researcher in the Hardware Devices group at Microsoft Research, Redmond, WA. Prior to joining Microsoft Research in March of 1998, he was an Institute Fellow at Georgia Tech and Director of the Interactive Media Technology Center. Prior to this, he was a founding engineer and manager of Future Products at IVEX Corporation, where he helped develop visual systems for flight simulation and ASICs for real-time image processing. He was also a research engineer at Georgia Tech's Research Institute and a toll terminal engineer at Western Electric Corporation. His main research interests are in user interface devices for the PC, PDAs, and wearables; microelectromechanical systems (MEMS) for interface applications; digital photography using linear sensors; and haptic interfaces. 